\title{
SOC Estimation Based on OCV for NiMH Batteries Using an Improved Takacs Model
}

\author{
Novie Ayub Windarko* and Jaeho Choi ${ }^{\dagger}$ \\ †* School of Electrical Eng., Chungbuk National University, Chungbuk, Korea
}

\begin{abstract}
This paper presents a new method for the estimation of State of Charge (SOC) for NiMH batteries. Among the conventional methods to estimate SOC, Coulomb Counting is widely used, but this method is not precise due to error integration. Another method that has been proposed to estimate SOC is by using a measurement of the Open Circuit Voltage (OCV). This method is found to be a precise one for SOC estimation. In NiMH batteries, the hysteresis characteristic of OCV is very strong compared to other type of batteries. Another characteristic of NiMH battery to be considered is that the OCV of a NiMH battery under discharging mode is lower than it is under charging mode. In this paper, the OCV is modeled by a simple method based on a hyperbolic function which well known as Takacs's model. The OCV model is then used for SOC estimation. Although the model is simple, the error is within $10 \%$.
\end{abstract}

Key Words: NiMH battery, OCV, SOC

\section{INTRODUCTION}

Nowadays, batteries are playing a significant role in energy storage applications. Due to environmental issues, battery applications in the energy saving and energy efficiency areas have become wide spread these days. So far, battery capacity in practical applications has reached up to hundreds $\mathrm{kWh}$ or several MWh. It has entered into practical applications such as hybrid electric vehicle (HEV), renewable energy [1], etc. In the near future, battery price will drop and then its application will be wider.

State-of-Charge (SOC) is used to determine battery capacity. In energy management systems, SOC monitoring has the following functions: It should prevent the battery from entering the modes of over-charge or over-discharge, since these conditions may damage the battery. In some applications, the SOC has to show users how long they can use a battery. For example, in HEV applications, the driver needs to know the residual driving range. The application of batteries for energy storage is often combined with Ultracapacitors. Batteries have high energy density and low power density. Ultracapacitors on the other hand, have low energy density and high power density. By combining both of these characteristics, they can maintain a power supply to meet any sudden change in load power demand. A PV system may need smoothing techniques due to output power variations [1]. This problem can be solved by applying a hybrid energy storage system composed of a high energy NiMH battery and an Ultracapacitors. This system

Manuscript received Nov. 12, 2009; revised Feb. 10, 2010

† Corresponding Author: choi@ @hungbuk.ac.kr

Tel: +82-43-261-2425, Fax: +82-43-276-7217, Chungbuk Nat'1 Univ.

* School of Electrical Eng., Chungbuk National University, Korea

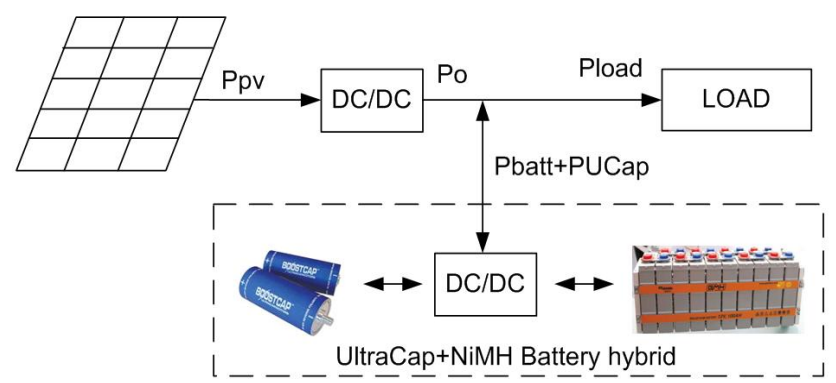

Fig. 1. Energy storage application in renewable energy systems.

configuration is shown in Fig.1. In this application, an Energy Management System including SOC monitoring is applied.

So far, Coulomb Counting method is the most popular method to estimate the SOC. Residual capacity is measured by a simple algorithm for the product of the current and the duration time of charge or discharge. This method can be more precise when it takes into consideration the temperature and the charging and discharging efficiencies [2]. On the other hand, it has the following disadvantages: There is no way to estimate the initial SOC and it depends on sensor precision. In addition, when these errors are integrated by the time, the result is high errors.

The measurement of open circuit voltage (OCV) is an accurate way to determine the SOC. OCV can be measured under the no load condition. This method is very suitable for estimating the initial SOC. However, under the charging or discharging state, the battery is not in the open circuit condition. Ideally, the OCV should be obtained after the battery has been at idle for more than ten hours [3]. In paper 


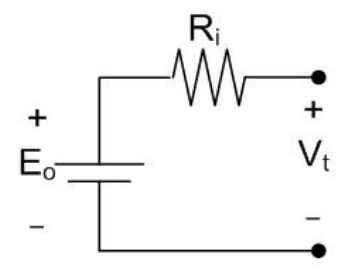

Fig. 2. Simple battery model.

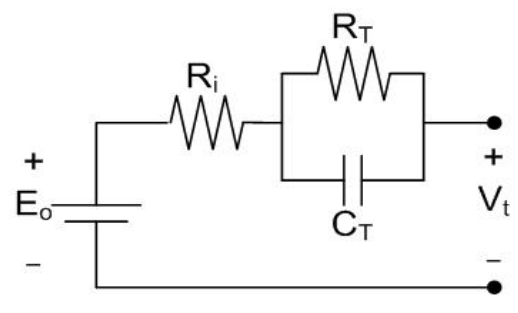

Fig. 3. Thevenin battery model.

[4], to avoid the long length of time required for observing the OCV, it is obtained by mathematical iteration.

These days, NiMH batteries are one of the most popular choices. These batteries have several advantages over other battery types, such as high energy density, low price, nontoxicity and safety. In a NiMH battery, the hysteresis characteristic of OCV is very strong when compared with other types. Another characteristic of NiMH batteries is that the OCV of these batteries under discharging mode is lower than it is under charging mode. The complexity of characteristics and its economic price are the challenges in the application of a NiMH battery management system (BMS).

This paper proposes a method to determine the SOC during the continuous charging or discharging processes, especially in $\mathrm{NiMH}$ batteries. Continuous processes are applied in several applications, such as: solar power, series type HEV, EV, etc. The SOC is estimated by the OCV. The OCV is estimate by a battery model showing the relationship between battery voltage, internal resistance and OCV. In this paper the OCV is modeled by a simple model based on a hyperbolic function which is well known as Takacs's model. The results of this modeling are compared with the experiment results.

\section{BATTERy MODELING}

\section{A. Electrical Modeling of Batteries}

The capacity of a battery is the product of the current and the duration of the charge or discharge. The SOC is determined by the original capacity minus the discharging capacity or plus the charging capacity. The SOC is expressed as:

$$
S O C_{t}=S O C_{0}-\int \eta \cdot I \cdot t d t
$$

where:

$S O C_{t}$ : SOC at time $t$

$S O C_{0}: S O C$ at the starting time

$\eta$ : discharging or charging efficiency

$I$ : battery current

$t$ : time

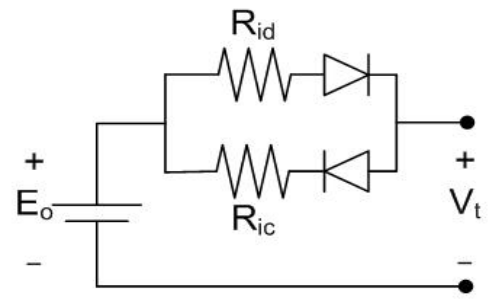

Fig. 4. Resistive Thevenin battery model.

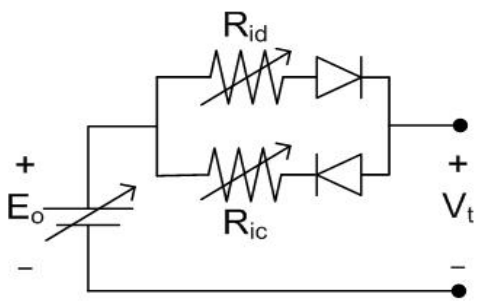

Fig. 5. Modified battery model.

Capacity can also be affected by temperature. The efficiency is influenced by internal resistance and other aspects such as temperature. Based on the definition in (1), the Coulomb counting method is very convenient although the precision of its modeling is influenced by several conditions as described previously.

Many battery models have been proposed. In this section the advantages and disadvantages of the conventional battery models are described. In addition, the modified resistive Thevenin battery model is proposed for which all elements are defined as a function of the SOC.

1) Simple Battery Model: This model is the most commonly used. As shown in Fig. 2. This model consists of the OCV or Electro Motive Force, $E_{o}$, and the series internal resistance, $R_{i}, V_{t}$ is the battery terminal voltage. $E_{o}$ is obtained from open circuit measurement. Although this model is commonly used, it cannot cover the varying characteristics of the internal resistance and OCV during the charging condition of the SOC. This model is only applicable in a simulation where the energy drawn from the battery is unlimited or the model element is independent from the SOC [5].

2) Thevenin battery Model: The Thevenin battery model is shown in Fig. 3 [6]. It consists of the OCV, $E_{o}$; the internal resistance, $R_{i}$; the RC parallel network of transient capacitance, $C_{T}$; the transient resistance, $R_{T}$. The added RC parallel circuit is for the prediction of a battery's response to transient load changes. In another paper [7], the RC parallel circuit is represented as a physical component, while $C_{T}$ is represented as parallel plates and $R_{T}$ is represented as a nonlinear resistance contributed from the contact resistance of the plate of the electrolyte. The disadvantage of this model is that the model elements are assumed to be constant despite the changing conditions of battery such as SOC, temperature, etc.

3) Resistive Thevenin Battery model: This model has two internal resistances based on the discharging and charging states as shown in Fig. 4. $R_{i c}$ and $R_{i d}$ are associated with the internal resistances under the charging mode and discharging mode, respectively. Both of these resistances are represented 


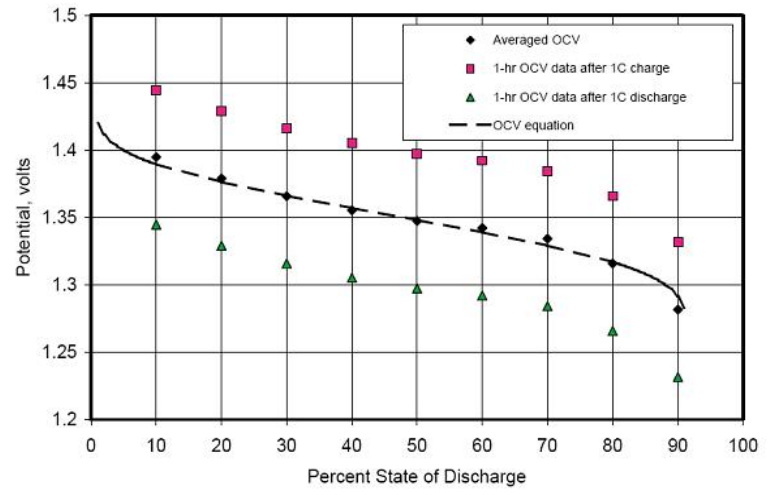

Fig. 6. Average OCV for charge and discharge [9].

by energy losses, which include both electrical and nonelectrical losses. In the diodes, it is implied that during the charging or discharging mode only one of the resistances $R_{i c}$ or $R_{i d}$ can be conducted. In the charging or discharging modes, the conducting diode is forward biased while the other diode is reversely biased. There is no physical meaning for the diodes without the modeling purposes. This model is not dependent on the SOC.

4) Proposed modified Model: In this paper, the authors propose a modified battery model as shown in Fig. 5. Generally, a battery model could be adopted from the Thevenin battery model. It uses the capacitor and resistor to explain the transient response during the charging/discharging response. In the case of a very long charging/discharging process time, the resistor-capacitor circuit reaches the steady state condition. In the steady state condition, only the resistor is considered and the capacitor can be ignored. In this model, all of the elements are defined as a function of the SOC. The OCV and resistor values are different from each other in the charging and discharging modes. In case of the charging mode, the OCV is higher than it is in the discharge mode.

The following equations are used for describing the model. Equation (2) describes the models of the charging and discharging modes, respectively.

$$
\begin{aligned}
& E_{o}(x)=V_{t}(x)-I_{c} R_{i c}(x) \\
& E_{o}(x)=V_{t}(x)+I_{d} R_{i d}(x)
\end{aligned}
$$

where $x$ is the variable for the SOC, and $I_{c}$ and $I_{d}$ are the charging and discharging currents, respectively. The elements of the model, $E_{o}, V_{t}$, and $R_{i}$ are obtained from the various currents of $I_{c}$ and $I_{d}$.

\section{B. Open Circuit Voltage Modeling}

1) Average OCV for Charging and Discharging Modes: Paper [8], describes the application of NiMH batteries to a HEV. The electrical model of the batteries is based on the Thevenin battery model with SOC dependence. The SOC monitoring algorithm is based on the average OCV during the charging or discharging modes. With a simple algorithm based on a simple average model, it is reported that a HEV can operate at the culminate efficiency of 80-mile-per-gallon. The dashed line in Fig. 6 is the averaged OCV regressed adaptively.
2) Preisach Model: The Preisach model has been used widely to estimate the model of hysteresis in many areas such as smart materials, sensors, actuators, electric-magnetic relays and transformers, etc. However, this model involves a complex calculation. In paper [9], the Preisach model is used for a HEV application. The continuous Preisach model was modified into a discrete model, which is more suitable for onboard hysteresis estimation due to the limited computational and memory resources. It is reported that the error of the algorithm is within $10 \%$ of the SOC.

3) Proposed OCV modeling based on an Improved Takacs Model: In this paper, authors propose the Takacs model for the description of the hysteresis phenomenon of OCV. The Takacs model is based on a mathematical function for hysteresis modeling [10]. The Takacs model follows several previous models based on mathematical models such as Langevin or Brillouin. Most of the hysteresis models are based on magnetic parameters such as magnetic flux density, magnetic field intensity, etc. The Takacs model with several parameters of the hysteresis curve is much simpler. The Takacs model parameters have no direct physical meaning in magnetic data experiments. For this reason, the Takacs model is suitable for the hysteresis modeling in batteries.

By using this model, the major and minor loop of the hysteresis curves can be modeled. In this paper, the major loop is discussed to verify the application of the Takacs model to describe the hysteresis characteristics of the relationship between the OCV and the SOC of a NiMH battery. In the Takacs model, the major loop can be divided into two branches, the ascending branch and the descending branch. The equations can be shown in (3):

$$
\begin{aligned}
& f_{+}(x)=\tanh \left(x-a_{0}\right)+b_{1} \\
& f_{-}(x)=\tanh \left(x+a_{0}\right)-b_{1}
\end{aligned}
$$

where $x$ is the variable for the OCV, $f_{+}$and $f_{-}$are the SOC in the charging and discharging states, respectively, $a_{o}$ is the initial SOC where $f_{+}(x)=0$, and $b_{1}$ is the value obtained from (4):

$$
b_{1}=\left[\tanh \left(x_{m}+a_{o}\right)-\tanh \left(x_{m}-a_{o}\right)\right] / 2
$$

where $x_{m}$ is the SOC at the maximum value of $f_{+}(x)$. Figure 7 shows the OCV modeling based on the original Takacs's model. It is obvious that the hysteresis curve obtained from the experimental data cannot be fully followed by this model. Therefore, authors propose an additional function to cover any of the hysteresis characteristics which are not fully followed by the original Takacs model. To improve the Takacs model so that it will match the hysteresis characteristics, the $f_{r}(x)$ term is added to the original Takacs model as shown in (5).

$$
\begin{aligned}
& f_{+}(x)=\tanh \left(x-a_{0}\right)+b_{1}+f_{+r}(x) \\
& f_{-}(x)=\tanh \left(x+a_{0}\right)-b_{1}+f_{-r}(x)
\end{aligned}
$$

where $f_{r}(x)$ is an error function between the measurement data and the original Takacs model. Since our motivation is to develop a simple model, $f_{r}(x)$ is proposed as a polynomial 


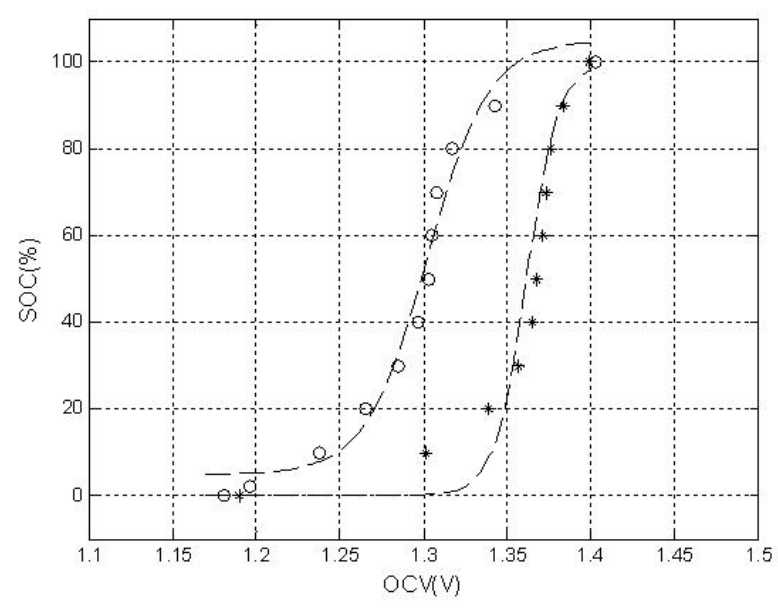

Fig. 7. Modeling of OCV vs SOC based on Takacs at $30^{\circ} \mathrm{C}$.

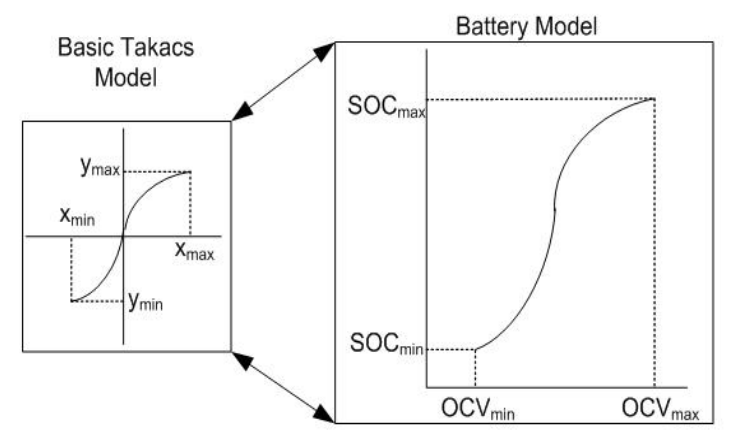

Fig. 8. Illustration for scaling technique.

function in this paper, which is expressed as:

$$
f_{r}(x)=c_{n} x^{n}+c_{n-1} x^{n-1}+\cdots+c_{1} x+c_{0}
$$

where $c_{0}, c_{1}, \cdots, c_{n}$ are the polynomial coefficients.

The Takacs model is based on a tangent hyperbolic function. A typical curve is shown in Fig. 8. As shown in this figure, the $x$-axis can be set from any value and the maximum value of the y-axis is unity. In a battery model, the $\mathrm{x}$-axis could be the OCV and the y-axis could be the SOC. The OCV value is higher than zero. For a single cell NiMH battery, the OCV is approximated to be $1.2 \sim 1.4 \mathrm{~V}$. The SOC value is in the range of $0 \sim 100 \%$. The difference between the original Takacs model and its application to the battery model can be solved by scaling. The scaling technique to solve the difference between the original Takacs model and the battery model is shown in Fig. 8 .

\section{RESULTS}

\section{A. Modeling}

In this experiment, a Sebang GMH 100 NiMH Battery rated at $1.2 \mathrm{~V}$ and $100 \mathrm{Ah}$ is used. The experiment is done at $30^{\circ} \mathrm{C}$. The experimental results are shown in Fig. 9 and Fig. 10. The OCV measurement for a $100 \%$ SOC is done under the charging mode with a charging current of $1 \mathrm{C}$. It is started at the empty capacity of $0 \%$ SOC where the battery voltage is $1.19 \mathrm{~V}$. The battery arrived at the full capacity of $100 \%$ SOC when the OCV, $V_{t}$ reached $1.52 \mathrm{~V}$. To measure the OCV, the

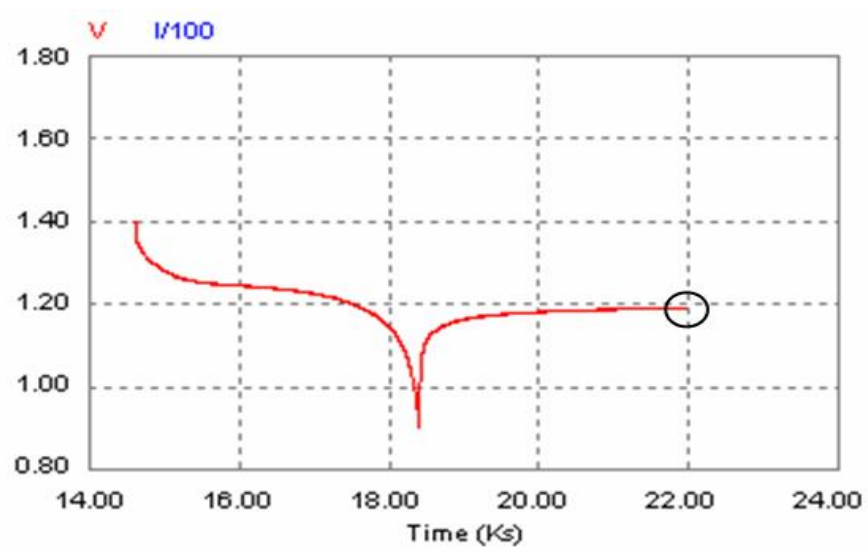

Fig. 9. OCV measurement after 1 hour rest for $100 \%$ SOC.

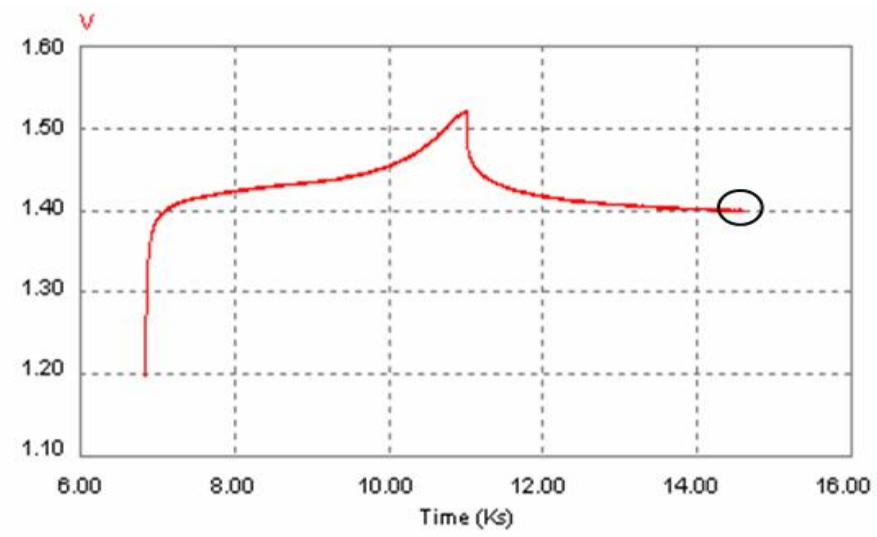

Fig. 10. OCV measurement after 1 hour rest for $0 \%$ SOC.

battery was allowed to rest for one hour. The meaning of 'rest' is that the battery is in the open circuit condition without any charging or discharging processes taking place. After one hour of rest, there is no significant change in the OCV. The change in OCV after one hour of rest is around $1 \mathrm{mV}$ or less. The $\mathrm{OCV}$ is obtained at the position indicated with a circle. At full capacity, the OCV is $1.403 \mathrm{~V}$.

The experiment on OCV measurement for a $0 \%$ SOC is shown in Fig. 10. It is done under the discharging mode with a discharging current of 1C. It is started at the full capacity of $100 \%$ SOC where the battery voltage is $1.403 \mathrm{~V}$. The battery arrives at the empty capacity of $0 \% \mathrm{SOC}$ when the OCV, $V_{t}$ is $0.9 \mathrm{~V}$. The $\mathrm{OCV}$ is obtained at the position indicated with a circle. At empty capacity, the OCV is $1.19 \mathrm{~V}$.

An OCV plot is shown in Fig. 11 [11]. The OCV is obtained for every $10 \%$ increase or decrease in the SOC. It is obviously that the OCV between a charging state and a discharging state is different. The non-linearity of the curve is very serious between $0-20 \%$ SOC and $80-100 \%$ SOC. To simplify the algorithm, several algorithms for a battery management system could be kept in the linear region of the SOC in the range of $20 \%-80 \%$. However, the disadvantage of this method is that it utilizes only $60 \%$ of the battery capacity [12]. As it was mentioned above, the improved Takacs model is proposed to cover the full range of the SOC. When the proposed model is used, the maximum error can be reduced to $10 \%$ in the full range of $0 \%-100 \%$. Near full or empty capacity, the 


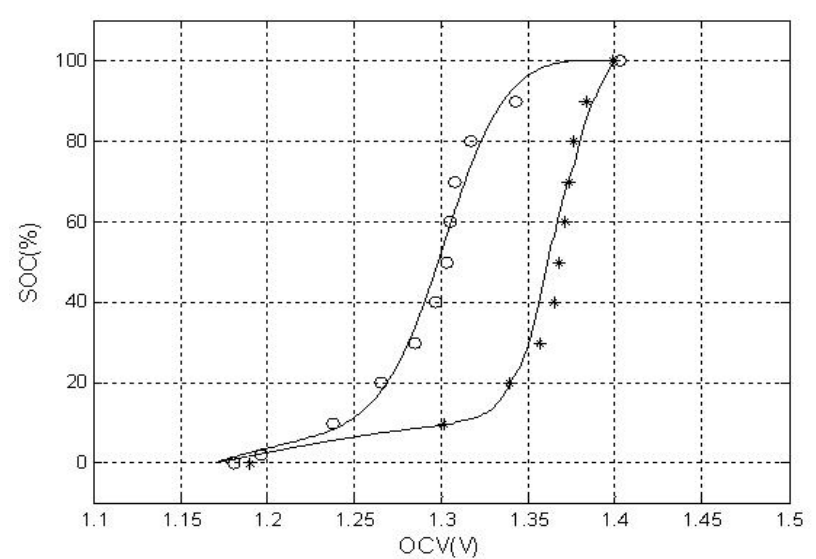

Fig. 11. Modeling of OCV vs SOC based on improved Takacs model at $30^{\circ} \mathrm{C}$.

error of the proposed modeling is close to zero. With this modeling, battery utilization can be increased to $100 \%$ SOC. The parameters of this model are shown in Table 1. The value of scale in Table 1 is the value of $x_{\min }$ and $x_{\max }$ of the basic Takacs model as shown in Fig. 8. By applying the scaling technique, the value of $b_{1}$ has no important meaning. As a result, $b_{1}$ can be ignored or the value can be zero.

The internal resistance in Fig. 12 is represented as a polynomial equation [11]. It is obtained from a calculation as shown in Eq. 2, where Eo or OCV, $V_{t}$, and $I$, are given by the measurements. The parameters for the polynomial equation are shown in Table 2.

The charging internal resistance, $R_{i c}$, is similar to a parabolic curve or a second order polynomial. Although the curve is similar to a parabolic curve, a fourth order polynomial is applied to reduce the error of the model.

The discharging internal resistance, $R_{i d}$, curve tends to be similar to a third order polynomial or odd function. However, $R_{i d}$ is modeled in the fourth order to reduce the error of the model. From Fig. 12, it should be noted that the low value of the resistance is correlated to high efficiency. In the case of the high efficiency operation mode, it should be operated at around 40\% SOC in charging mode. In discharging mode, the internal resistance is low in a high SOC.

\section{B. Temperature Effect}

The temperature dependency of the OCV-SOC is shown in Fig. 13. Note that the curve shown for the temperature dependency in Fig. 13 is from $0^{\circ} \mathrm{C}$ to $45^{\circ} \mathrm{C}$. It is shown that lower temperature operation increases the $\mathrm{OCV}$ value. Around the linear region of the curve, the OCV values are similar. At a higher or lower SOC, the differences in OCV values are increased. The differences between the OCVs measured at different temperatures are shown in Fig. 14. The OCV at $30^{\circ} \mathrm{C}$ is used as a reference. The differences in OCV in Fig. 14 can be expressed by:

$$
O C V_{d}=O C V_{t^{\circ} \mathrm{C}}-O C V_{30^{\circ} \mathrm{C}} .
$$

Note that the OCV in Eq. 7 has the same value of SOC. The $O C V_{d}$ between $0^{\circ} \mathrm{C}$ and $30^{\circ} \mathrm{C}$ is indicated by a triangle

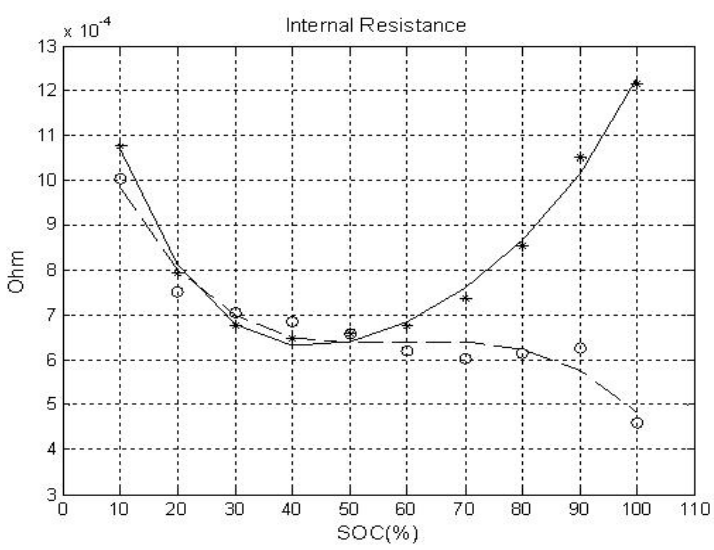

Fig. 12. Discharging (०) and charging(*) internal resistance vs SOC at $30^{\circ} \mathrm{C}$.

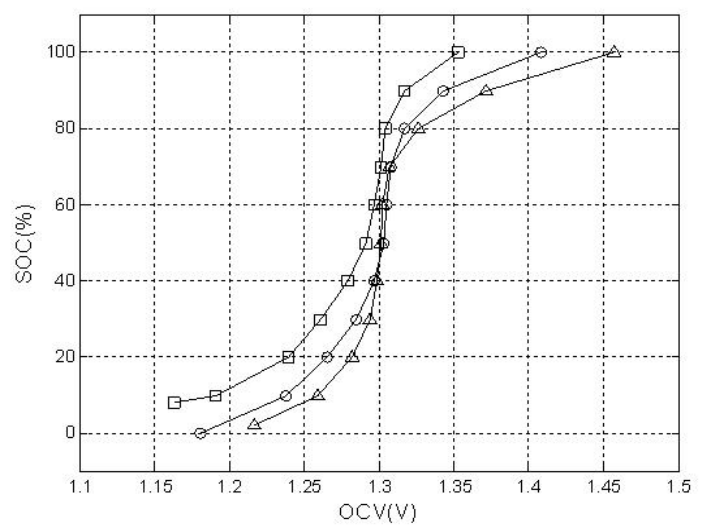

Fig. 13. Temperature effect to OCV in discharging mode. ( $\triangle=0^{\circ} \mathrm{C}$, $\left.\bigcirc=30^{\circ} \mathrm{C}, \square=45^{\circ} \mathrm{C}\right)$

$(\triangle)$, and between $45^{\circ} \mathrm{C}$ and $30^{\circ} \mathrm{C}$ it is indicated by a square ( $\square$ ). The maximum difference in OCV is $55 \mathrm{mV}$. It occurred at $100 \%$ SOC at $45^{\circ} \mathrm{C}$. As shown in Fig. 13, at around $20 \%$ to $80 \%$ SOC, the curve is linear. The slope in the linear region is high. This means that a small difference in the SOC will cause larger errors in the SOC. The difference in the OCV in the linear region could result in a SOC error up to $20 \%$. To reduce this error, a compensation function could be applied for the battery operating at any temperature. The compensation function, $f_{c}$, could be adopted based on the OCV difference curve as shown in Fig. 14. The curve of the compensation function is similar to a parabolic function, which can be modeled by a simple second order polynomial or higher.

Based on the previous explanation, an algorithm for SOC estimation can be developed to:

1) Obtain the OCV and battery temperature.

2) Convert the OCV from step 1 to the $\mathrm{OCV}$ at $t=30^{\circ} \mathrm{C}$ by a compensation function.

3) Estimate the SOC from the OCV from step 2 by using the OCV-SOC curve at $t=30^{\circ} \mathrm{C}$.

\section{CONCLUSIONS}

An estimation method for the SOC of NiMH batteries based on the OCV is proposed. The model is proposed for the continuous charging or discharging modes. The method 


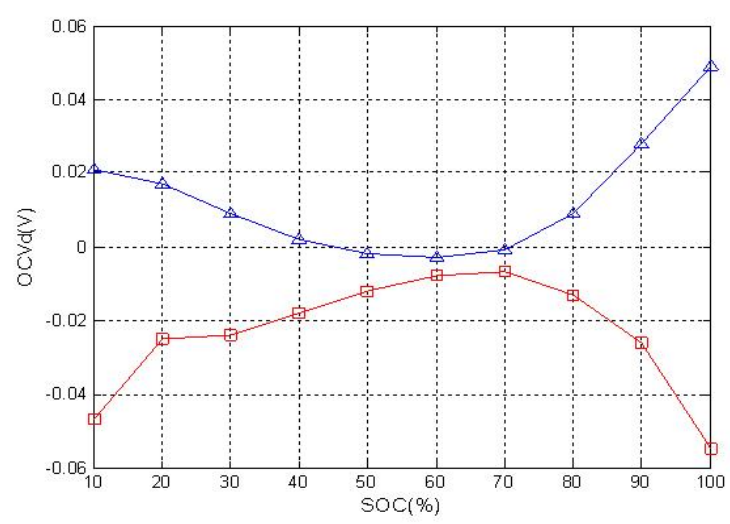

Fig. 14. Difference of $\mathrm{OCV}$ at different temperature between $0^{\circ} \mathrm{C}$ and $30^{\circ} \mathrm{C}(\triangle)$ and between $45^{\circ} \mathrm{C}$ and $30^{\circ} \mathrm{C}(\square)$.

is especially for $\mathrm{NiMH}$ batteries in which the hysteresis of $\mathrm{OCV}$ is very strong. The OCV between the charging and discharging modes is different. Based on the internal resistance curve, it should be considered the operation range if the high efficiency operation is needed. The experimental data on battery operation in different temperatures show that the OCV value is higher at lower temperatures. The OCV modeling in continuous mode can be useful for applications in solar power, series type $\mathrm{HEV}, \mathrm{EV}$, etc.

\section{APPENDIX}

TABLE I

MODELING PARAMETERS FOR OCV

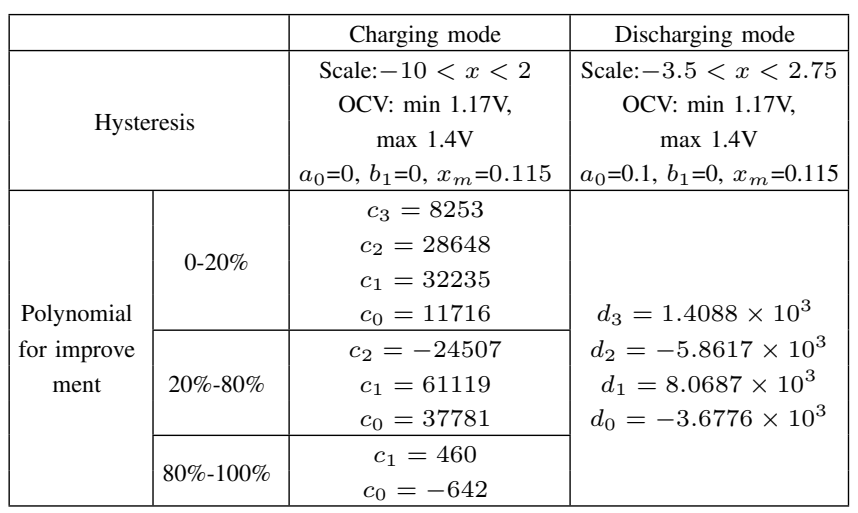

TABLE II

MODELING PARAMETERS FOR INTERNAL RESISTANCE

\begin{tabular}{|c|c|c|c|c|c|}
\hline & $C_{4}$ & $C_{3}$ & $C_{2}$ & $C_{1}$ & $C_{0}$ \\
\hline \multirow{2}{*}{ Charge } & 41.94 & -10.61 & 1.14 & -53.09 & 1.49 \\
& $\times 10^{-12}$ & $\times 10^{-9}$ & $\times 10^{-6}$ & $\times 10^{-6}$ & $\times 10^{-6}$ \\
\hline \multirow{2}{*}{ Discharge } & 5.95 & -4.20 & 621.16 & -33.81 & 1.26 \\
& $\times 10^{-12}$ & $\times 10^{-9}$ & $\times 10^{-9}$ & $\times 10^{-6}$ & $\times 10^{-3}$ \\
\hline
\end{tabular}

\section{ACKNOWLEDGMENT}

This research was supported by the MKE (Ministry of Knowledge Economy), Korea, under the ITRC (Information
Technology Research Center) support program supervised by the IITA (Institute for Information Technology Advancement) (IITA-2009-C1090-0904-0007)

\section{REFERENCES}

[1] Manoj Datta, T. Senjyu, A. Yona, H. Sekine, and T. Funabashi, "Smoothing output power variations of isolated utility connected multiple PV systems by coordinated control," Journal of Power Electronics, Vol.9, No.2, pp. 320-333, Mar. 2009.

[2] Kong Soon Ng, Chin-Sien Moo, Yi-Ping Chen, and Yao-ching Hsieh, "Enhanced coulomb counting method for estimating state-of-charge and state-of-health of litihium-ion batteries," Journal of Applied Energy, Vol. 86, No. 9, pp. 1506-1511, Sep. 2009.

[3] Wu Guoliang, Lu Rengui, Zhu Chunbo, and CC Chan, "State of charge estimation for NiMH battery based on electromotive force method," Conf. Proc. IEEE Vehicle Power and Propulsion, pp.1-5, Sep. 2008.

[4] Antoni Szumanowski and Yuhua Chang, "Battery management system based on battery nonlinear dynamics modeling," IEEE Trans on Vehicular Tech, Vol. 57, No. 3, pp. 1425-1432, May 2008.

[5] Salameh et. Al, "A mathematical model for lead-acid batteries," IEEE Trans. on Energy Conversion, Vol. 7, No.1, pp. 93-97, Mar. 1992.

[6] Min Chen and Gabriel A. Rincon-Mora, "Accurate electrical battery model capable of predicting runtime and I-V performance," IEEE Trans. on Energy Conversion, Vol. 21, No. 2, pp. 504-511, Jun. 2006.

[7] D Sutanto and HL Chang, "A new battery model for use with battery energy storage systems and electric vehicles power systems," Conf. Proc. of IEEE Power Engineering Society Winter Meeting, Vol. 1, pp. 470-475, Jan. 2000.

[8] Mark Verbrugge and Edward Tate, "Adaptive state of charge algorithm for nickel metal hydride batteries including hysteresis phenomena," $J$. of Power Sources, Vol. 126, No. 1-2, pp. 236-249, Feb. 2004.

[9] Xidong Tang, Xiaodong Zhang, Brian Koch, and Damon Frisch, "Modeling and estimation of nickel metal hydride battery hysteresis for SOC estimation," Conf. Proc. of Prognostics and Health Management, pp. $1-12,2008$.

[10] J. Takacs, "A phenomenological mathematical model of hysteresis," Int. J. for Computation and Mathematics in Electrical and Electronics Engineering, Vol. 20, No. 4, pp. 1022-1014, 2001.

[11] Novie Ayub Windarko, Jaeho Choi, and Deoksu Hyun, "SOC estimation based on OCV for continuous charging/discharging process in NiMH battery," Proc. of $2^{\text {nd }}$ Japan-Korea Workshop Joint Technical Workshop on Semiconductor Power Converter, pp. 1-6, 2009.

[12] Wei Li and Geza Joos, "A power electronic interface for a battery supercapacitor hybrid energy storage systems for wind applications," Conf. Proc. of IEEE PESC 2008, pp. 1762-1768, Jun. 2008.

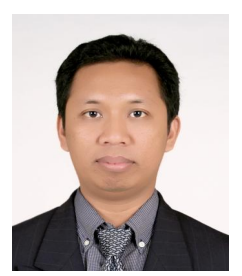

Novie Ayub Windarko received his B.E and M.E in Electrical Engineering from Sepuluh Nopember Institute of Technology, Surabaya Indonesia, in 1999 and 2006 respectively. He has been with the Electronic Engineering Polytechnic Institute of Surabaya, Indonesia, since 2000. He was a Visiting Researcher in the Akagi Lab., Tokyo Institute of Technology, Japan, from 2002 to 2003. Currently, he is working towards his $\mathrm{Ph}$. $\mathrm{D}$. at the School of Electrical Engineering, Chungbuk National University, Korea. His research interests are in power electronics, power quality, energy storage systems and renewable energy.

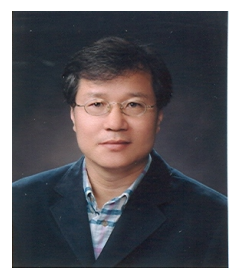

Jaeho Choi received his B.S, M.S. and Ph.D. in Electrical Engineering from Seoul National University, Seoul, Korea in 1979, 1981 and 1989, respectively. From 1981 to 1983, he was with the Jungkyong Technical College, Daejeon, Korea as a Full-time Lecturer. Since 1983, he has been with the School of Electrical Engineering, Chungbuk National University in Cheongju, Korea, where he is currently a Professor. In 1993, 1998, 2003 and 2009, he was a Visiting Professor at the University of Toronto in Canada each for one year and he was a Danfoss Visiting Professor at the Aalborg University in Denmark in 2000. His research interests include power electronics, power quality problems and solutions, energy storage systems and renewable energy and microgrid systems. He is an active member of KIEE, KIPE and IEEE and he is currently the Editor-in-Chief of the Journal of Power Electronics. 